\begin{tabular}{|c|c|}
\hline Title & Control of plasma CVD SiO $2 / \mathrm{nA}$ IN interface by $\mathrm{N} 20$ plasma oxidation \\
\hline Author(s) & A kazawa, Masamichi; Kitajima, Shouhei; Kitawaki, Y uya \\
\hline Citation & $\begin{array}{l}\text { Japanese Journal of A pplied Phy sics (JJAP), 58(10), } 106504 \\
\text { https://doi .org/ } 10.7567 / 1347-4065 / \text { ab3c49 }\end{array}$ \\
\hline Issue Date & 2019-10-01 \\
\hline Doc URL & http:/hdl.handle.net/2115/79367 \\
\hline Rights & (C) 2019 The Japan Society of A pplied Physics \\
\hline Type & article (author version) \\
\hline File Information & JJA P-101707_for printing.pdf \\
\hline
\end{tabular}

Instructions for use 


\title{
Control of plasma-CVD $\mathrm{SiO}_{2} /$ InAIN interface by $\mathrm{N}_{2} \mathrm{O}$ plasma oxidation
}

\author{
Masamichi Akazawa*, Shouhei Kitajima and Yuya Kitawaki \\ Research Center for Integrated Quantum Electronics, Hokkaido University, Sapporo \\ 060-0813, Japan \\ E-mail: akazawa@rciqe.hokudai.ac.jp
}

Control of the plasma-CVD $\mathrm{SiO}_{2} / \mathrm{InAlN}$ interface by $\mathrm{N}_{2} \mathrm{O}$ plasma oxidation of the InAlN surface was studied. The interface was characterized by both X-ray photoelectron spectroscopy (XPS) and capacitance-voltage measurement of metal-insulatorsemiconductor (MIS) diodes. An excessively long duration of oxidation led to the deterioration of the stoichiometry of the InAIN surface and plasma oxide, resulting in a high density of interface states in the completed MIS diodes. Meanwhile, the surface-localized oxygen deficiency in the plasma oxide layer was observed by XPS. The intensity ratio of the oxygen-deficient component to the fully oxidized component in the $\mathrm{O} 1 \mathrm{~s}$ spectrum decreased with increasing oxidation duration. Consequently, there was an optimum oxidation duration. The interface state density was reduced by almost one order in the case of plasma oxidation for an appropriate duration compared with the case of the direct deposition of $\mathrm{SiO}_{2}$ onto InAlN. 


\section{Introduction}

$\mathrm{GaN}$ has attracted much attention as a material for developing power electronics because of its wide band gap, high saturation electron velocity, high breakdown field, and good thermal conductivity. ${ }^{1)}$ One of the advantages of III-nitrides is their feasibility for constructing excellent heterostructures. ${ }^{1)}$ As an application to high-frequency power devices, $\mathrm{AlGaN} / \mathrm{GaN}$ heterostructures providing a high-mobility and high-density two-dimensional electron gas (2DEG) are used to construct high-electron-mobility transistors (HEMTs). $\mathrm{In}_{x} \mathrm{Al}_{1-x \mathrm{~N}} \mathrm{~N}$, which achieves lattice matching to $\mathrm{GaN}$ when $x=0.17-0.18$, is a promising alloy for HEMT application because it can provide a higher-density $2 \mathrm{DEG}^{2}$ ) owing to its high-density spontaneous polarization ${ }^{3)}$ and large conduction band offset ${ }^{4)}, \Delta E_{\mathrm{C}}$, with a large band gap. ${ }^{5)}$ Actually, a 2DEG density higher than $2 \times 10^{13} \mathrm{~cm}^{-2}$ has been reported. ${ }^{6-11)}$ However, InAlN has the drawback of a high leakage current ${ }^{12)}$ owing to the high internal electric field resulting from the high spontaneous polarization. ${ }^{13)}$ Therefore, the use of an insulator for the gate structure is necessary. So far, several insulators, i.e., $\mathrm{Al}_{2} \mathrm{O}_{3}{ }^{12}{ }^{14-18)}$, $\mathrm{ZrO}_{2}{ }^{15,19,20)}, \mathrm{GdScO}_{3}{ }^{15)}, \mathrm{HfO}_{2}{ }^{19)}, \mathrm{SiO}_{2}{ }^{21)}$, plasma oxides ${ }^{22-26)}$, and thermal oxides ${ }^{27,28)}$, have been used to construct insulated gate HEMTs or metal-insulator-semiconductor (MIS) HEMTs. Among these insulators, $\mathrm{SiO}_{2}$ has the widest band gap, achieving a large $\Delta E_{\mathrm{C}}$ with InAlN lattice-matched to GaN. Since the insulator/semiconductor interface is generally characterized by the presence of interface states, which deteriorate the device performance, it is desirable to establish a method of controlling the $\mathrm{SiO}_{2} / \mathrm{InAlN}$ interface.

In our previous work ${ }^{29)}$, we reported that $\mathrm{N}_{2} \mathrm{O}$ plasma oxidation of an InAlN surface is a promising method of reducing the interface state density $D_{\text {it }}$ at the $\mathrm{SiO}_{2} / \mathrm{InAlN}$ interface. However, the optimum conditions for surface oxidation and the mechanism underlying the reduction in $D_{\text {it }}$ have not been clarified. Recently, plasma oxidation of the III-nitride surface has been reported to be an excellent method of achieving surface passivation of $A 1 G a N / G a N$ HEMTs. ${ }^{30)}$ In addition, the existence of an ultrathin epitaxial crystalline intermediate $\mathrm{Ga}_{2} \mathrm{O}_{3}$ layer at the plasma-CVD $\mathrm{SiO}_{2} / \mathrm{GaN}$ interface has been reported, suggesting that the initial oxidation of the GaN surface leads to good interfacial properties. ${ }^{31)}$ These results indicate that the properties of the native oxide/III-nitride interface formed by plasma oxidation are excellent. However, the reason why the plasma-induced damage does not deteriorate the interface properties is unclear. Generally, ion attacks cause plasma damage at the sample 
surface, resulting in interface disorder with random changes in the connection, length, and angle of the chemical bonds at the interface. The direct deposition of $\mathrm{SiO}_{2}$ onto an InAlN surface by plasma CVD leads to the formation of an uncontrolled oxide layer, resulting in the generation of interface states. ${ }^{29}$ ) On the other hand, successful control of the insulator/AlGaN interface by $\mathrm{N}_{2} \mathrm{O}$ radical treatment while excluding ion attacks has been reported. ${ }^{32)}$ Therefore, an excellent native oxide/III-nitride interface can be formed only when plasma oxidation is performed appropriately. To realize this, the mechanism of formation of an excellent native oxide/III-nitride interface by plasma oxidation should be investigated.

In our very recent work ${ }^{33)}$, we reported that inserting a 0.5 -nm-thick $\mathrm{Al}_{2} \mathrm{O}_{3}$ interlayer formed by atomic layer deposition (ALD) at a plasma-CVD $\mathrm{SiO}_{2} / \mathrm{InAlN}$ interface reduces $D_{\text {it }}$ efficiently. On the basis of X-ray photoelectron spectroscopy (XPS) results, it has been clarified that the native oxide is formed beneath the ultrathin $\mathrm{Al}_{2} \mathrm{O}_{3}$ interlayer. Therefore, the reduction in $D_{\text {it }}$ should have been achieved by forming an excellent native oxide/InAlN interface with ion attacks blocked by the ultrathin $\mathrm{Al}_{2} \mathrm{O}_{3}$ interlayer during plasma CVD. There is a possibility that the native oxide layer grown by plasma oxidation can automatically block ion attacks at the interface.

In this work, the control of the plasma-CVD $\mathrm{SiO}_{2} / \mathrm{InAlN}$ interface by $\mathrm{N}_{2} \mathrm{O}$ plasma oxidation of the InAlN surface prior to $\mathrm{SiO}_{2}$ deposition was investigated. On the basis of XPS results, the oxide growth rate, stoichiometry of the InAlN surface and plasma oxide, and oxygen deficiency in the plasma oxide were analyzed. From the results of capacitancevoltage $(C-V)$ measurement of MIS diodes combined with simulation results, $D_{\text {it }}$ was extracted. An appropriate duration of plasma oxidation led to a reduction in $D_{\text {it }}$ compared with the case of the direct deposition of $\mathrm{SiO}_{2}$ by plasma CVD, whereas a very long duration of oxidation resulted in an increase in $D_{\text {it. }}$ The mechanism of $D_{\text {it }}$ reduction at the optimum duration is discussed on the basis of the combined results of XPS and electrical measurement.

\section{Experimental procedure}

For electrical measurements, MIS diodes, as shown in Fig. 1, were fabricated as follows. A Si-doped InAlN layer was grown by metal organic vapor phase epitaxy (MOVPE) on 
sapphire substrates via a GaN buffer layer. The carrier concentration $n$ of the InAlN layer was adjusted to $1-3 \times 10^{18} \mathrm{~cm}^{-3}$ and the thickness was $160 \mathrm{~nm}$, which enabled us to characterize the samples as ordinary MIS diodes without considering the 2DEG that was located much deeper than the depletion layer edge. (Although we have not performed any special characterizations, the quality of the 160-nm-thick InAlN epitaxial layer was satisfactory for use in a MIS structure to characterize insulator-semiconductor interfaces by the $C-V$ method and to compare the effects of fabrication process.) Chemical treatment was carried out using buffered hydrofluoric acid (HF) solution $\left(\mathrm{BHF}, \mathrm{NH}_{4} \mathrm{~F}: \mathrm{HF}=5: 1\right)$ to remove the surface oxide. A 20-nm-thick SiN layer to protect the InAlN surface during ohmic annealing was deposited by electron cyclotron resonance plasma CVD at $270{ }^{\circ} \mathrm{C}$ with $100 \mathrm{~W}$ plasma power using a $\mathrm{N}_{2}$ and $\mathrm{SiH}_{4} / \mathrm{Ar}$ gas mixture. After photolithography and partial removal of SiN using BHF, a Ti $(20 \mathrm{~nm}$; bottom)/Al $(50 \mathrm{~nm}) / \mathrm{Ni}(20 \mathrm{~nm}) / \mathrm{Au}(50$ $\mathrm{nm}$; topmost) ohmic contact was formed as a large pad perforated with a periodic-hole array. Subsequently, the sample with the SiN surface protection layer was annealed at 850 ${ }^{\circ} \mathrm{C}$ for $1 \mathrm{~min}$. After annealing, the SiN layer was removed using the BHF solution. Then the sample surface was oxidized using $\mathrm{N}_{2} \mathrm{O}$ RF plasma at $20 \mathrm{~W}$ plasma power, a substrate temperature of $300{ }^{\circ} \mathrm{C}$, and a pressure of $28 \mathrm{~Pa}$ in a $\mathrm{N}_{2} \mathrm{O}$ flow of $27 \mathrm{sccm}$. The durations of plasma oxidation were set at $30 \mathrm{~s}, 1 \mathrm{~min}$, and $3 \mathrm{~min}$. For comparison, a sample without plasma oxidation was also fabricated. $\mathrm{SiO}_{2}$ was deposited by $\mathrm{RF}$ plasma $\mathrm{CVD}$ at $300{ }^{\circ} \mathrm{C}$ and $30 \mathrm{~W}$ plasma power using a $\mathrm{N}_{2} \mathrm{O}$ and $\mathrm{SiH}_{4} / \mathrm{Ar}$ gas mixture. The thickness of the $\mathrm{SiO}_{2}$ layer was in the range of 21-25 nm. Finally, a Ni (20 nm, lower)/Au (50 nm, upper) circular gate electrode was formed at the center of the hole of the ohmic contact pad to complete the MIS structure.

The plasma oxide/InAlN interface was examined by XPS using a sample with an ultrathin plasma oxide layer formed on an undoped 30-nm-thick InAlN layer grown by MOVPE on a sapphire substrate via a $2-\mu \mathrm{m}$-thick GaN buffer layer. A monochromated Al-Ka X-ray source (1486.6 eV) was used. The charge-up error in binding energy was calibrated by setting the $\mathrm{C} 1 \mathrm{~s}$ spectral peak to $285.0 \mathrm{eV}$.

\section{Results and discussion}

The XPS In $3 \mathrm{~d}, \mathrm{~N} 1 \mathrm{~s}$, and A1 2p spectra observed at the photoelectron take-off angle $\theta$ of 
$45^{\circ}$ for the InAlN surface after plasma oxidation for various durations are shown in Fig. 2. Oxide components are observed clearly in the Al $2 \mathrm{p}$ and In $3 \mathrm{~d}$ spectra, whereas no oxide component can be seen in the $\mathrm{N}$ 1s spectrum. The shape of the $\mathrm{N}$ 1s spectrum obtained from the InAlN surface after plasma oxidation coincided with that of the HF-treated InAlN surface. Therefore, a native oxide layer without oxynitride components was formed by this plasma oxidation. It can be seen that the intensity ratio $R_{\mathrm{I}}$ of the oxide component to the InAlN component increased with the oxidation duration in both the In $3 \mathrm{~d}$ and $\mathrm{Al} 2 \mathrm{p}$ spectra. This indicates that the thickness of the surface oxide layer increased with the oxidation duration. Here, the oxide thickness can be estimated from the obtained Al $2 p$ spectrum as follows. The integrated intensity ICL InAIN of a core-level spectrum from the InAIN layer decays with increasing plasma oxide thickness $d$ POX as ${ }^{34)}$

$I_{C L I n A l N}=I_{0 C L I n A l N} \exp \left(-\frac{d_{P O X}}{\lambda}\right)$,

where $I_{0 \mathrm{CL}}$ InAIN indicates $I_{\mathrm{CL} \text { InAIN }}$ with $d_{\mathrm{POX}}=0$, and $\lambda$ is the escape depth of photoelectrons given by

$\lambda=\lambda_{0} \sin \theta$

where $\lambda_{0}$ is the inelastic mean free path of photoelectrons. Using $\lambda_{0}$ determined by the theoretical calculation ${ }^{35,36)}$, we calculated $\lambda$ at $\theta=45^{\circ}$ to be $1.7,1.8$, and $2.2 \mathrm{~nm}$ for $\mathrm{In} 3 \mathrm{~d}$, $\mathrm{N} 1 \mathrm{~s}$, and $\mathrm{Al} 2 \mathrm{p}$, respectively. On the other hand, the integrated intensity ICL POX of the same core-level spectrum from the plasma oxide layer increases with an increase in $d$ POX $\mathrm{as}^{34)}$

$I_{C L P O X}=I_{0 C L \text { POX }}\left\{1-\exp \left(-\frac{d_{P O X}}{\lambda}\right)\right\}$

where $I_{0 \mathrm{CL}}$ POX indicates $I_{\text {CL POX }}$ with an infinite or sufficiently large $d$ POX. Consequently, for the spectra obtained from the oxidized InAlN surface, $R_{\mathrm{I}}$ is given by ${ }^{34)}$ 
$R_{I}=\left(\frac{I_{0 C L P O X}}{I_{0 C L I n A l N}}\right) \frac{1-\exp \left(-\frac{d_{P O X}}{\lambda}\right)}{\exp \left(-\frac{d_{P O X}}{\lambda}\right)}$.

Using this equation, $d$ POX was derived from $R_{\mathrm{I}}$. Here, we used the Al $2 \mathrm{p}$ spectrum because the ratio $I_{0 \mathrm{CL}}$ POX/I0CL InAIN can be extracted from the $\mathrm{Al}_{2} \mathrm{O}_{3} / \mathrm{InAlN}$ structures formed by ALD, with the assumption that the molar fraction of $\mathrm{Al}$ oxide in the native oxide layer is equal to that of AIN in InAIN. The extracted $d_{\text {POX }}$ is plotted as a function of oxidation duration in Fig. 3. It can be seen that $d_{\text {POX }}$ increases with the oxidation duration. The dashed line in Fig. 3 shows an example fitting line calculated with the Deal-Grove model $^{37)}$ given by

$d_{P O X}=\frac{A}{2}\left\{\left(1+\frac{t+\tau}{A^{2} / 4 B}\right)^{1 / 2}-1\right\}$

where $A$ and $B$ are used as fitting parameters (an explanation of their physical meaning is omitted here) and $\tau$ is a parameter related to the initial thickness and was set to 0 .

Figure 4 shows the integrated intensity $I_{\mathrm{CL} \text { InAIN }}$ as a function of the extracted $d_{\mathrm{POX}}$ for the In $3 \mathrm{~d}, \mathrm{~N} 1 \mathrm{~s}$, and $\mathrm{Al} 2 \mathrm{p}$ spectra. The dashed lines indicate fitting curves given by Eq. (1). Although reasonable fitting within the experimental error can be seen for $\mathrm{N} 1 \mathrm{~s}$ and $\mathrm{Al} 2 \mathrm{p}$, the discrepancy between the estimated intensity and the measured intensity is considerable for In $3 \mathrm{~d}$ at the largest $d$ POX. It is highly likely that the stoichiometry at the InAIN surface was deteriorated by the oxidation for $3 \mathrm{~min}$. In addition, the stoichiometry of the plasma oxide layer was also deteriorated by 3 min oxidation. The intensity ratio $R_{\mathrm{I}}$ for the $\mathrm{Al} 2 \mathrm{p}$ and In $3 \mathrm{~d}$ core-level spectra is plotted as a function of $d_{\mathrm{POX}}$ in Fig. 5, where the solid and dashed lines are fitting lines calculated by Eq. (4). It can be seen that the measured $R_{\mathrm{I}}$ for In $3 \mathrm{~d}$ deviates from the estimation at the largest $d$ POX, being much lower than the estimated value. This result indicates a deficit of the In component in the plasma oxide layer for the sample with $3 \mathrm{~min}$ oxidation. On the basis of these results, it is concluded that an excessively long duration of oxidation leads to deterioration of the stoichiometry of the InAlN surface and plasma oxide. Presumably, the In component at the InAlN surface is more difficult to oxidize compared with $\mathrm{Al}$ component, or the $\mathrm{Al}$ component is more easily oxidized than the In component, when the oxidation duration is too long. This phenomenon 
should affect the quality of the insulator/semiconductor interface of the completed MIS diodes.

The chemical bond connection in the native oxide layer may also affect the interface quality. $\mathrm{O} 1 \mathrm{~s}$ spectra obtained at $\theta=45^{\circ}$ for different oxidation durations are illustrated in Fig. 6(a), where the O 1s spectra are decomposed into two components, in which the Inand Al-related components are merged for each, with a chemical shift of $\sim 1.5 \mathrm{eV}$. The lower-energy component denoted by $\mathrm{O}_{\mathrm{I}}$ consists of $\mathrm{In}_{2} \mathrm{O}_{3}{ }^{38)}$ and $\mathrm{Al}_{2} \mathrm{O}_{3}{ }^{39)}$. It is reported that the hydroxide components exhibits higher binding energy with a similar chemical shift. ${ }^{38}$, 39) However, since the $\mathrm{N}_{2} \mathrm{O}$ oxidation was carried out in vacuum, it is unlikely that hydroxides dominate in the higher-energy component denoted by OII. Alternatively, it is highly likely that the higher-energy component is mainly composed of the oxygen-deficient region including dangling bonds ${ }^{40-42}$. The spatial location of this region was clarified by angle-resolved XPS, as shown in Fig. 6(b), where O 1s spectra observed at $\theta=15,45$, and $90^{\circ}$ are plotted for the 1-min-oxidized sample. It can be clearly seen that the intensity of the $\mathrm{O}_{\text {II }}$ component increased as $\theta$ decreased. Since a decrease in $\theta$ makes the analysis more surface-sensitive; this result indicates that the oxygen-deficient region was localized at the topmost surface of the native oxide layer. On the other hand, in Fig. $6(a)$, it can be seen that the ratio of the integrated intensity of the oxygen-deficient component IoII to that of the fully oxidized component IOI decreased with increasing oxidation duration. This ratio $\left(I_{\mathrm{OII}} / I_{\mathrm{OI}}\right)$ is plotted in Fig. 7 as a function of $d_{\mathrm{POX}}$, where its almost linear decay can be seen. The effect of this tendency is discussed later.

The XPS results described above should be related to the electrical properties of the interface. It is naturally expected that the quality of the $\mathrm{SiO}_{2} / \mathrm{InAlN}$ interface of the completed MIS diode is dependent on the native oxide/InAlN interface. One of the most important parameters concerning the interface quality is $D_{\text {it. }}$ According to the disorder-induced gap state (DIGS) model $^{43}$, interface disorder is the origin of interface states. Since the stoichiometry of the InAlN surface and plasma oxide deteriorated in the sample with 3 min plasma oxidation, there is a possibility that interface disorder was introduced at the interface. Therefore, it is anticipated that $D_{\text {it }}$ of the MIS diodes with 3 min plasma oxidation is high. Figure 8 shows a summary of the results of the $C-V$ measurement at a frequency of $1 \mathrm{MHz}$, where the solid lines indicate the measured data, 
the one-dot-chain lines are the calculated ideal curves, and the open circles indicate simulated curves used to fit the measured ones. For comparison, the data taken from Ref. 33 for the sample with direct $\mathrm{SiO}_{2}$ deposition are also plotted in Fig. 8(a). The oxide thicknesses were $20 \mathrm{~nm}$ for direct $\mathrm{SiO}_{2}$ deposition, $25 \mathrm{~nm}$ for the 30-s- and 3-min-oxidized samples, and $21 \mathrm{~nm}$ for the 1-min-oxidized sample. The difference in $\mathrm{SiO}_{2}$ thickness was due to an error in thickness control. The doping density also varied among the sample owing to the unoptimized doping conditions. Nevertheless, the properties of the samples can be compared using the ideal curves, which were calculated by taking into account the difference in the conditions, as a benchmark. It can be seen that the sample with 1 min plasma oxidation exhibits the best $C-V$ characteristic, whereas that of the sample with 3 min plasma oxidation indicates the existence of a high $D$ it. The ideal $C-V$ curves are calculated using a previously developed simulator. ${ }^{44,45)}$ Generally, the measured curve exhibits a voltage shift from the ideal curve. In Fig. 8, the ideal curves were shifted in the voltage axis direction to overlap with the measured $C-V$ curves for comparison. The magnitude of this voltage shift of each ideal $C-V$ curve is denoted by $\Delta V$ and summarized in Table I. The origin of $\Delta V$ is discussed later.

Using the same simulator, $C-V$ curves were calculated to obtain the best fit to the measured data by taking into account the charge/discharge of interface states. The $D_{\text {it }}$ distribution was assumed to be U-shaped on the basis of the DIGS model. ${ }^{43)}$ As a mathematical model of the $D_{\text {it }}$ distribution, the following equation can be appropriately used to simulate the $C-V$ curves $^{44,45)}$ :

$$
D_{i t}(E)=D_{i t 0} \exp \left(\left|\frac{E-E_{C N L}}{E_{0 D, 0 A}}\right|^{n_{D}, n_{A}}\right)
$$

where $D_{\mathrm{it} 0}$ is the minimum of $D_{\mathrm{it}}, E_{\mathrm{CNL}}$ is the charge neutrality level, $E_{0 \mathrm{D}}$ and $n_{\mathrm{D}}$ are the curvature factors assumed for the donorlike states below $E_{\mathrm{CNL}}$, and $E_{0 \mathrm{~A}}$ and $n_{\mathrm{A}}$ are those for the acceptorlike states above $E_{\mathrm{CNL}}$. $E_{\mathrm{CNL}}$ was assumed to be located $2.0 \mathrm{eV}$ below the conduction band minimum energy, $E_{\mathrm{C}}{ }^{17)}$. Therefore, $E_{0 \mathrm{D}}$ and $n_{\mathrm{D}}$ cannot be determined from the $C-V$ curves of the n-type MIS diode because the response of the interface states located below $E_{\mathrm{CNL}}$ becomes too slow. Here, $D_{\mathrm{it} 0}, E_{0 \mathrm{~A}}$, and $n_{\mathrm{A}}$ are used as parameters for fitting to the measured $C-V$ curves. By fitting, we can extract the $D_{\text {it }}$ distributions. The extracted $D_{\text {it }}$ 
distributions are summarized in Fig. 9, where the data for the sample with the direct $\mathrm{SiO}_{2}$ deposition are taken from our previous work ${ }^{33)}$. It can be seen that the 1-min-oxidized sample exhibited the lowest $D_{\text {it, }}$ whereas the 3-min-oxidized sample showed the highest $D_{\text {it. }}$ Compared with the sample without plasma oxidation, the sample with the most appropriate duration of oxidation achieved a one-order reduction in $D_{\text {it. }}$.

In our previous work ${ }^{33)}$, we argued that $\Delta V$ is the sum of the polarization charge $Q_{\text {pol, }}$ the fixed charge $Q_{\mathrm{f}}$ distributed at the interface and possibly in insulator, and the charge $Q_{i t}^{f r}$ owing to the frozen interface states with a long time constant compared with the speed of the bias sweep. However, using $Q_{i t}^{f r}$ determined from the $D_{\text {it }}$ distributions extracted by the simulation, the extracted $Q_{\mathrm{f}}$ was very high even for the $\mathrm{SiO}_{2} / \mathrm{InAlN}$ interface controlled by the $\mathrm{Al}_{2} \mathrm{O}_{3}$ interlayer in Ref. 33 and that controlled by the plasma oxide interlayer in this work, resulting in a high $Q_{\mathrm{f}} / q$ on the order of $10^{13} \mathrm{~cm}^{-2}$ ( $q$ is the elemental charge). Since this density seems to be too high for an excellent interface with a low $D_{\text {it, }}$, the estimation of $Q_{\mathrm{f}} / q$ should be reappraised. Because $Q_{\mathrm{pol}} / q$ is as large as $-2.88 \times 10^{13} \mathrm{~cm}^{-2}$ ) , an estimation of $Q_{\mathrm{f}} / q$ results in a high value. There is a possibility that another type of charge compensated for $Q_{\text {pol }}$ at the interface or the InAlN surface. One of the possible compensation charges is surface donors ${ }^{46)}$. If this charge is taken into account, $\Delta V$ is given by

$$
\Delta V=-\frac{1}{C_{I}}\left(Q_{p o l}+Q_{S D}+Q_{f}+Q_{i t}^{f r}\right)
$$

where $C_{\mathrm{I}}$ is the insulator capacitance and $Q_{\mathrm{SD}}$ is the charge density of surface donors. Nevertheless, the exact value of $Q_{\mathrm{SD}}$ is unclear here. Therefore, we can only obtain an apparent fixed charge $Q_{\text {fapp }}$ given by

$$
Q_{f a p p}=Q_{p o l}+Q_{S D}+Q_{f}
$$

In the simulation, this $Q_{\text {fapp }}$ and the $D_{\text {it }}$ distribution were adjusted as parameters to fit the $C$ $V$ curves. Namely, the $D_{\text {it }}$ distribution that determined $Q_{i t}^{f r}$ was adjusted to reproduce the shape of the $C-V$ curve, whereas $Q_{\text {fapp }}$ was adjusted so that the simulated curve overlapped 
the measured curve, resulting in the determination of $\Delta V$ given by Eq. (7). Figure 10 plots the extracted $Q_{\text {fapp }} / q$ as a function of $d$ POX. It can be seen that $Q_{\text {fapp }}$ increases with plasma oxide interlayer thickness. This indicates that the plasma oxide interlayer contains the bulk fixed charge, although the origin of this bulk charge is unclear at this stage.

On the basis of the results of both XPS and electrical measurements, the optimum oxidation duration was found. An excessively long duration of oxidation resulted in the deterioration of the stoichiometry of the InAlN surface and plasma oxide, which led to interface disorder that generated a high $D_{\text {it. }}$ On the other hand, shorter durations of $30 \mathrm{~s}$ and

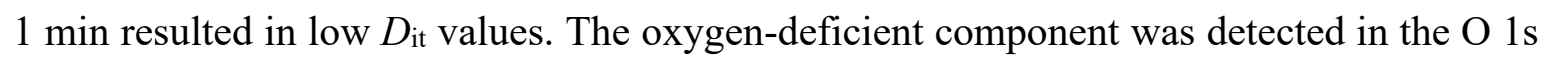
spectra of all samples, as shown in Fig. 6(a), although IoI//IOI decreased with increasing oxidation duration as shown in Fig. 7. This means that the oxygen-deficient component existed in the native oxide layer even in the 1-min-oxidized sample with the lowest $D_{\text {it. }}$ It seems unusual that an interface with oxygen deficiency exhibited a low $D_{\text {it. }}$ The decrease in $I_{\mathrm{OII}} / I_{\mathrm{OI}}$ with increasing oxidation duration in Fig. 7 was likely to be due to the increase in thickness, which led to a decrease in the ratio of the surface-localized component to the oxide-bulk component. There is a possibility that the oxygen-deficient region is the product of plasma damage that introduces defects and/or dangling bonds at the topmost surface of the native oxide layer, whereas a plasma oxide layer without oxygen deficiency or any defects exists at the native oxide/InAlN interface. That is, it is highly likely that plasma oxide prevents the native oxide/InAIN interface from ion attacks by itself. Considering that the lowest $D_{\text {it }}$ was observed in 1-min-oxidized sample, the amount of the surface-localized OII component might have been reduced after $\mathrm{SiO}_{2}$ deposition owing to chemical bonding at the $\mathrm{SiO}_{2}$ /native oxide interface, achieving a reduction in $D_{\mathrm{it}}$ in the completed MIS structure. Nevertheless, since residual defects might have existed in the vicinity of the $\mathrm{SiO}_{2} /$ native oxide interface and affected the interface states at the native oxide/InAlN interface by tunneling, the thickness of the native oxide layer should be sufficiently large to reduce $D_{\text {it }}$ by separating these interfaces. The slight increase in $D_{\text {it }}$ of the 30 -s-oxidized sample possibly resulted from its slightly smaller plasma oxide interlayer thickness, or higher tunneling probability, than that of the 1-min-oxidized sample. Consequently, a tradeoff exists between maintaining the stoichiometry of the InAIN surface and plasma oxide and separating the two interfaces, which results in an optimum oxidation duration. 


\section{Conclusions}

Control of the plasma-CVD $\mathrm{SiO}_{2} / \mathrm{InAlN}$ interface by $\mathrm{N}_{2} \mathrm{O}$ plasma oxidation of the InAlN surface was studied. The interface was characterized by both XPS and $C-V$ measurement of MIS diodes subjected to $30 \mathrm{~s}, 1 \mathrm{~min}$, and $3 \mathrm{~min}$ interface oxidation. The $3 \mathrm{~min}$ oxidation led to the deterioration of the stoichiometry of the InAlN surface and plasma oxide, resulting in a high density of interface states in the completed MIS diode. On the other hand, the lowest $D_{\text {it, }}$ which was almost one order lower than that of the sample with the direct deposition of $\mathrm{SiO}_{2}$, was achieved by 1 min plasma oxidation. The surface-localized oxygen-deficient component in the plasma native oxide layer was observed in the XPS O 1s spectrum. The intensity ratio of the oxygen-deficient component to the fully oxidized component decreased with increasing the oxidation duration and resultant oxide thickness. A slight increase in $D_{\text {it }}$ was obtained for the 30-s-oxidized sample compared with the 1-min-oxidized sample, which is possibly because of the slightly smaller native oxide thickness.

\section{Acknowledgments}

This work was supported by JSPS KAKENHI Grant Number JP15K04672 and JP16H06421. 


\section{References}

1) D. Ueda, in Power GaN Devices, eds. M. Meneghini, G. Meneghesso, and E. Zanoni (Springer, Berlin, 2017) Chap. 1.

2) J. Kuzmík, IEEE Electron. Device Lett. 22, 510 (2001).

3) O. Ambacher, R. Dimitrov, M. Stutzmann, B. E. Foutz, M. J. Murphy, J. A. Smart, J. R. Shealy, N. G. Weimann, K. Chu, M. Chumbes, B. Green, A. J. Sierakowski, W. J. Schaff and L. F. Eastman, Phys. Status Solidi B 216, 381 (1999).

4) W. Walukiewicz, S. X. Li, J. Wu, K. M. Yu, J. W. Ager III, E. E. Haller, H. Lu, and W. J. Schaff, J. Cryst. Growth 269, 119 (2004).

5) R. E. Jones, R. Broesler, K. M. Yu, J. W. Ager III, E. E. Haller, W. Walukiewicz, X. Chen and W. J. Schaff, J. Appl. Phys. 104, 123501 (2008).

6) M. Higashiwaki and T. Matsui, Jpn. J. Appl. Phys. 43, L768 (2004).

7) J. Kuzmík, A. Kostopoulos, G. Konstantinidis, J.-F. Carlin, A. Georgakilas and D. Pogany, IEEE Electron. Device Lett. 53, 422 (2006).

8) M. Gonschorek, J.-F. Carlin, E. Feltin, M. A. Py and N. Grandjean, Appl. Phys. Lett. 89, 062106 (2006).

9) J. Xie, X. Ni, M. Wu, J. H. Leach, Ü. Özgür and H. Morkoç, Appl. Phys. Lett. 91, 132116 (2007).

10) M. Gonschorek, J.-F. Carlin, E. Feltin, M. A. Py, N. Grandjean, V. Darakchieva, B. Monemar, M. Lorenz and G. Ramn, J. Appl. Phys. 103, 093714 (2008).

11) L. Ardaravičius, M. Ramonas, J. Liberis, O. Kiprijanovič, A. Matulionis, J. Xie, M. Wu, J. H. Leach and H. Morkoç, J. Appl. Phys. 106, 073708 (2009).

12) G. Pozzovivo, J. Kuzmík, S. Golka, W. Schrenk, G. Strasser, D. Pogany, K. Ĉiĉo, M. Ťapajna, K. Fröhlich, J.-F. Carlin, M. Gonschorek, E. Feltin and N. Grandjean, Appl. Phys. Lett. 91, 043509 (2007).

13) J. Kotani Atsushi Yamada, Tetsuro Ishiguro, Shuichi Tomabechi and Norikazu Nakamura, Appl. Phys. Lett. 108, 152109 (2016).

14) K. Čičo, J. Kuzmík, J. Liday, K. Hušeková, G. Pozzovivo, J.-F. Carlin, N. Grandjean, D. Pogany, P. Vogrinčič and K. Fröhlich, J. Vac. Sci. Technol. B27, 218 (2009).

15) K. Čičo, K. Hušeková, M. Ťapajna, D. Gregušová, R. Stoklas, J. Kuzmík, J.-F. Carlin, N. Grandjean, D. Pogany and K. Fröhlich, J. Vac. Sci. Technol. B29, 01A808 (2011). 
16) M. Ťapajna, K. Čičo, J. Kuzmík, D. Pogany, G. Pozzovivo, G. Strasser, J.-F. Carlin, N. Grandjean and K. Fröhlich, Semicond. Sci. Technol. 24, 035008 (2009).

17) M. Akazawa, M. Chiba and T. Nakano, Appl. Phys Lett. 102, 231605 (2013).

18) S. Ozaki, K. Makiyama, T. Ohki, N. Okamoto, S. Kaneki, K. Nishiguchi, N. Hara, and T. Hashizume, Appl. Phys. Express 10, 061001 (2017).

19) J. Kuzmík, G. Pozzovivo, S. Abermann, J.-F. Carlin, M. Gonschorek, E. Feltin, N. Grandjean, E. Bertagnolli, G. Strasser and D. Pogany, IEEE Trans. Electron Devices 55, 937 (2008).

20) P. Kordoš, R. Stoklas, D. Gregušová, K. Hušeková, J.-F. Carlin and N. Grandjean, Appl. Phys. Lett. 102, 063502 (2013).

21) M. Lachab, M. Sultana, Q. Fareed, F. Husna, V. Adivarahan and A. Khan, J. Phys. D: Appl. Phys. 47, 135108 (2014).

22) D. S. Lee, X. Gao, S. Guo and T. Palacios, IEEE Electron Device Lett. 32, 617 (2011).

23) D. S. Lee, J. W. Chung, H. Wang, X. Gao, S. Guo, P. Fay and T. Palacios, IEEE Electron Device Lett. 32, 755 (2011).

24) D. S. Lee, X. Gao, S. Guo, D. Kopp, P. Fay and T. Palacios, IEEE Electron Device Lett. 32, 1525 (2011).

25) Y. Yue, Z. Hu, J. Guo, B. Sensale-Rodriguez, G. Li, R. Wang, F. Faria, T. Fang, B. Song, X. Gao, S. Guo, T. Kosel, G. Sinder, P. Fay, D. Jena and H. Xing, IEEE Electron Device Lett. 33, 988 (2012).

26) Y. Yue, Z. Hu, J. Guo, B. Sensale-Rodriguez, G. Li, R. Wang, F. Faria, B. Song, X. Gao, S. Guo, T. Kosel, G. Sinder, P. Fay, D. Jena and H. Xing, Jpn. J. Appl. Phys. 52, 08JN14 (2013).

27) M. Eickelkamp, M. Weingarten, L. Rahimzadeh Khoshroo, N. Katteniss, H. Behmenburg, M.Heuken, D. Donoval, A. Chvála, P. Kordoš, H. Kalisch and A. Vescan, J. Appl. Phys. 110, 084501 (2011).

28) S. Ozaki, K. Makiyama, T. Ohki, Y. Kamada, M. Sato, Y. Niida, N. Okamoto and K. Joshin, Phys. Status Solidi A 213, 1259 (2015).

29) M. Akazawa and A. Seino, Phys. Status Solidi B 254, 1600691 (2017).

30) M. Mi, X.-H. Ma, L. Yang, Y. Lu, B. Hou, J. Zhu, M. Zhang, H.-S. Zhang, Q. Zhu, L.-A. Yang, and Y. Hao, IEEE Trans. Electron. Devices 64, 4875 (2017). 
31) K. Mitsuishi, K. Kimoto, Y. Irokawa, T. Suzuki, K. Yuge, T. Nabatame, S. Takashima, K. Ueno, M. Edo, K. Nakagawa, and Y. Koide, Jpn. J. Appl. Phys. 56, 110312 (2017).

32) Y. Hori, Z. Yatabe, and T. Hashizume, J. Appl. Phys. 114, 244503 (2013).

33) M. Akazawa and S. Kitajima, Jpn. J. Appl. Phys. 58, SIIB06 (2019).

34)D. Briggs and M. P. Seah, Practical Surface Analysis by Auger and X-ray Photoelectron Spectroscopy (John Wiley \& Sons Ltd., Sussex, 1983) Chap. 4.

35) S. Tanuma, C. J. Powell, and D. R. Penn, Surf. Interface Anal. 17, 927 (1991).

36) S. Tanuma, C. J. Powell, and D. R. Penn, Surf. Interface Anal. 21,165 (1993).

37) E. H. Nicollian and J. R. Brews, MOS (Metal Oxide Semiconductor) Physics and Technology (John Wiley \& Sons, Inc., Hoboken, 1981) Chap. 13.

38) N. Asai, Y. Inoue, H. Sugimura, and O. Takai, J. Electrochem. Soc. 146, 2365 (1999).

39) M. R. Alexander, G. E. Thompson and G. Beamson, Surf. Interface Anal. 29, 468 (2000).

40) J. S. Kim, P. K. H. Ho, D. S. Thomas, R. H. Friend, F. Cacialli, G.-W. Bao, and S. F. Y. Li, Chem. Phys. Lett. 315, 307 (1999).

41) A. Thøgersen, M. Rein, E. Monakhov, J. Mayandi, and S. Diplas, J. Appl. Phys. 109, 113532 (2011).

42) Y. Gao, J. Lu, J. Zhang, and X. Li, J. Alloys Compd. 646, 675 (2015).

43) H. Hasegawa and H. Ohno, J. Vac. Sci. \& Technol. B 4, 1130 (1986).

44) M. Miczek, C. Mizue, T. Hashizume and B. Adamowicz, J. Appl. Phys. 103, 104510 (2008).

45) M. Miczek, B. Adamowicz, C. Mizue and T. Hashizume, Jpn. J. Appl. Phys. 48, 04C092 (2009).

46) M. Ťapajna and J. KuzmíK, Appl. Phys. Lett. 100, 113509 (2012). 


\section{Figure Captions}

Fig. 1. Structure of tested MIS diode.

Fig. 2. XPS spectra obtained from InAlN surface after plasma oxidation, i.e., In $3 \mathrm{~d}, \mathrm{~N} 1 \mathrm{~s}$, and $\mathrm{Al} 2 \mathrm{p}$ spectra for oxidation durations of $30 \mathrm{~s}, 1 \mathrm{~min}$, and $3 \mathrm{~min}$.

Fig. 3. Plot of the thickness of the oxide layer formed by plasma oxidation, derived from the intensity ratio of the oxide component to the InAlN component in the Al $2 p$ spectrum, as a function of oxidation duration.

Fig. 4. Plots of integrated intensity $I_{\mathrm{CL}}$ InAIN as a function of $d \mathrm{POX}$. The dashed lines are fitting lines calculated by Eq. (1) assuming $\lambda$ to be 1.7, 1.8, and $2.2 \mathrm{~nm}$ for In $3 \mathrm{~d}, \mathrm{~N} 1 \mathrm{~s}$, and $\mathrm{Al} 2 \mathrm{p}$, respectively.

Fig. 5. Plots of integrated intensity ratio $R_{\mathrm{I}}$ of oxide component to InAlN component as a function of $d \mathrm{POX}$ for $\mathrm{Al} 2 \mathrm{p}$ and In $3 \mathrm{~d}$ core level spectra. The dashed and solid lines indicate the theoretical estimation calculated by Eq. (4) for Al $2 p$ and $\mathrm{In} 3 \mathrm{~d}$, respectively.

Fig. 6. (a) $\mathrm{O} 1 \mathrm{~s}$ spectra obtained at $\theta=45^{\circ}$ for oxidation durations of $30 \mathrm{~s}, 1 \mathrm{~min}$, and 3 min. (b) $\mathrm{O}$ 1s spectra obtained by angle-resolved XPS for the 1-min-oxidized InAlN surface.

Fig. 7. Plot of intensity ratio $I_{\mathrm{OII}} / I_{\mathrm{OI}}$ as a function of $d \mathrm{POX}$.

Fig. 8. $C-V$ curves measured at $1 \mathrm{MHz}$ for fabricated MIS diodes (solid lines) with the calculated ideal curves (one-dot-chain lines) and the simulated curves (open circles). (a) Direct $\mathrm{SiO}_{2}$ deposition (Data are taken from Ref. 33). (b) $\mathrm{N}_{2} \mathrm{O}$ oxidation for 30 s. (c) $\mathrm{N}_{2} \mathrm{O}$ oxidation for $1 \mathrm{~min}$. (d) $\mathrm{N}_{2} \mathrm{O}$ oxidation for $3 \mathrm{~min}$.

Fig. 9. Summary of the $D_{\text {it }}$ distributions assumed in the simulation for the best fit to the measured $C-V$ curves. The data for the sample with direct $\mathrm{SiO}_{2}$ deposition are taken from 
Ref. 33.

Fig. 10. Plot of $Q_{\text {fapp }} / q$ as a function of plasma oxide interlayer thickness. The solid circles indicate $Q_{\text {fapp }} / q$ estimated for the MIS diodes, and the solid line is the fitting line to the solid circles. 
Table I. Summary of $\Delta V$ for the ideal curves.

\begin{tabular}{cc}
\hline Oxidation duration [min] & $\Delta V[\mathrm{~V}]$ \\
\hline 0 & 3.2 \\
0.5 & 2.8 \\
1.0 & 2.1 \\
3.0 & 2.8 \\
\hline
\end{tabular}

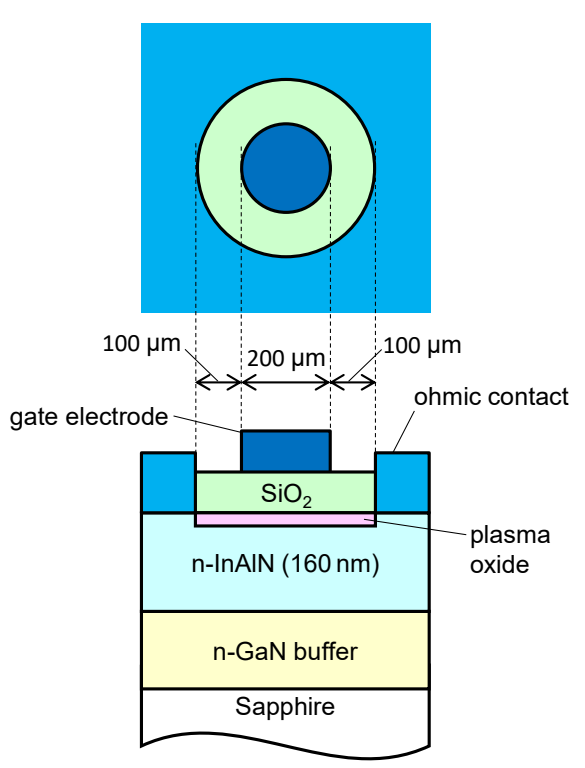

Fig. 1 


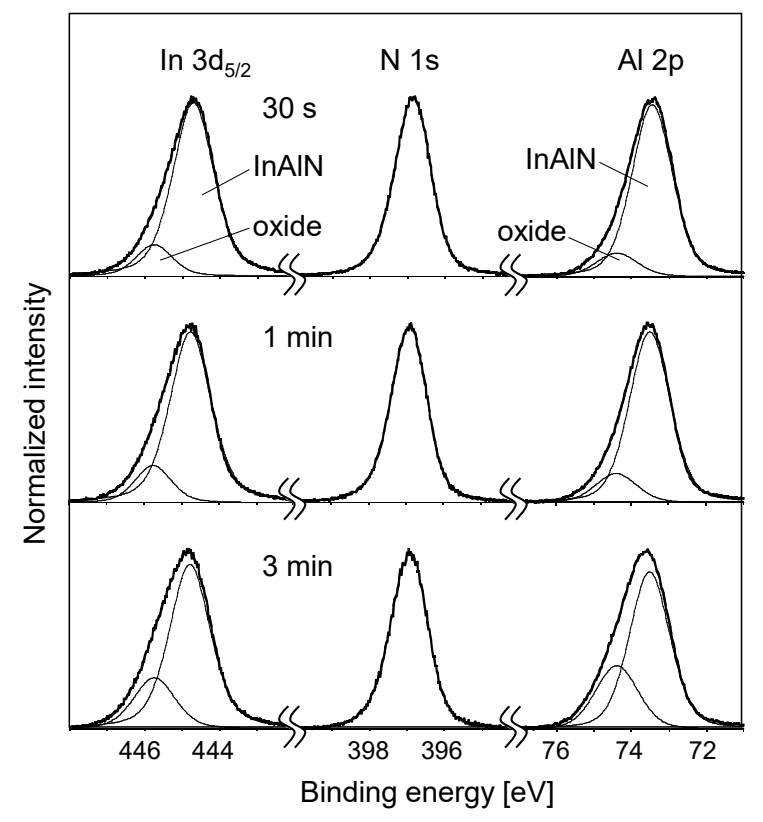

Fig. 2

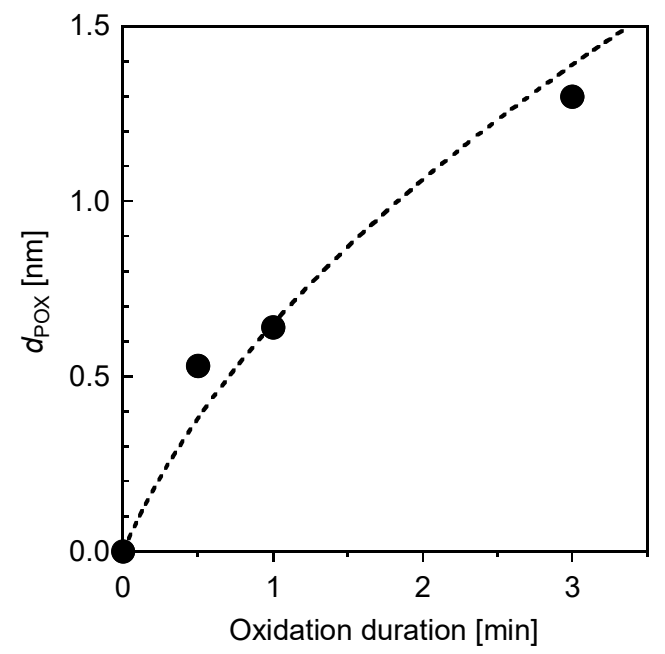

Fig. 3 


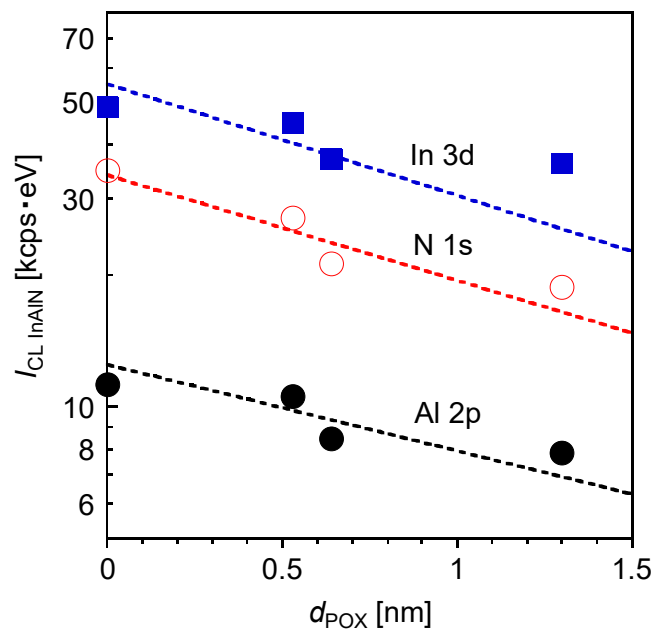

Fig. 4

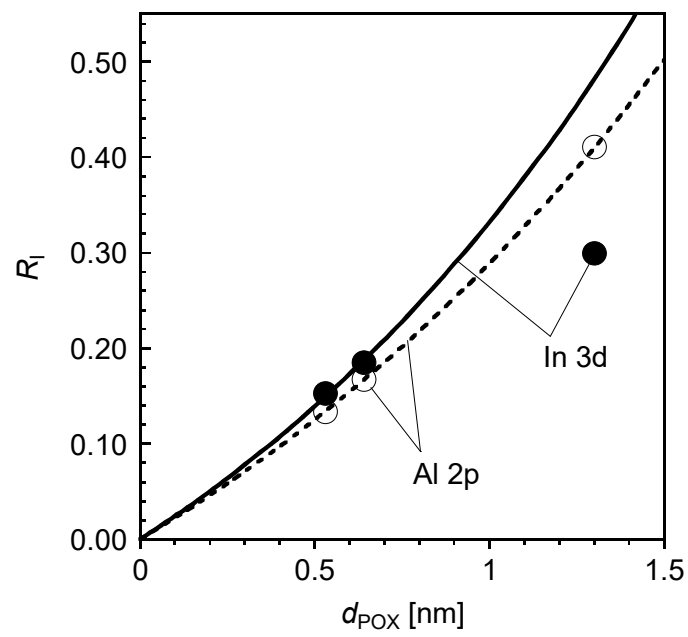

Fig. 5 

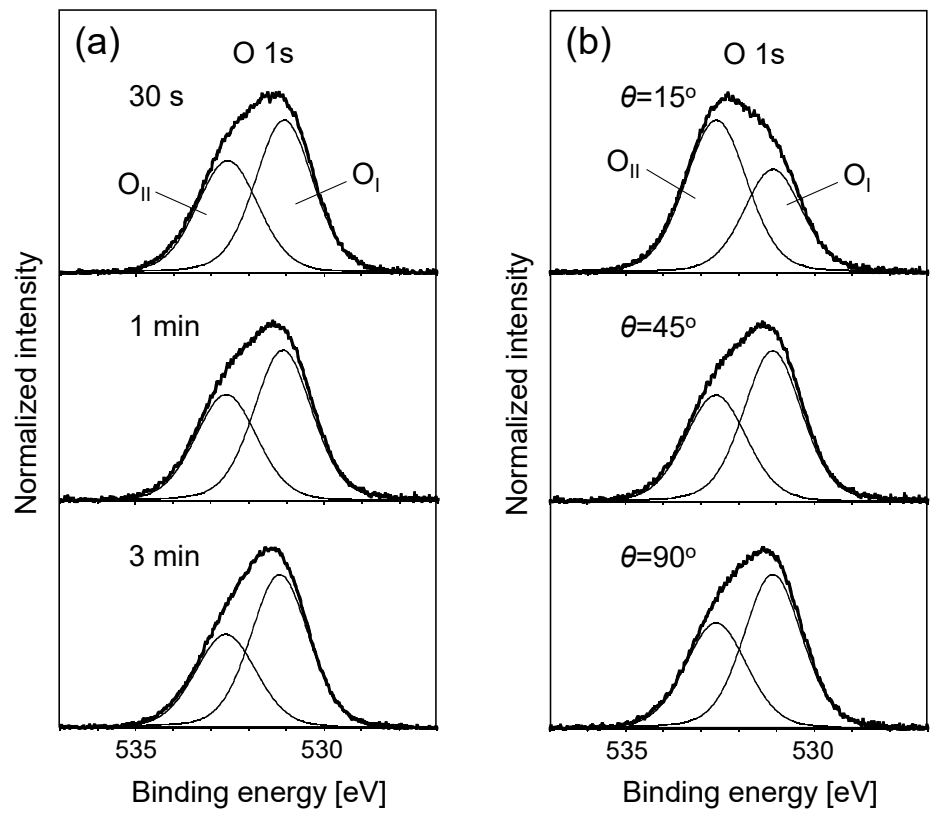

Fig. 6

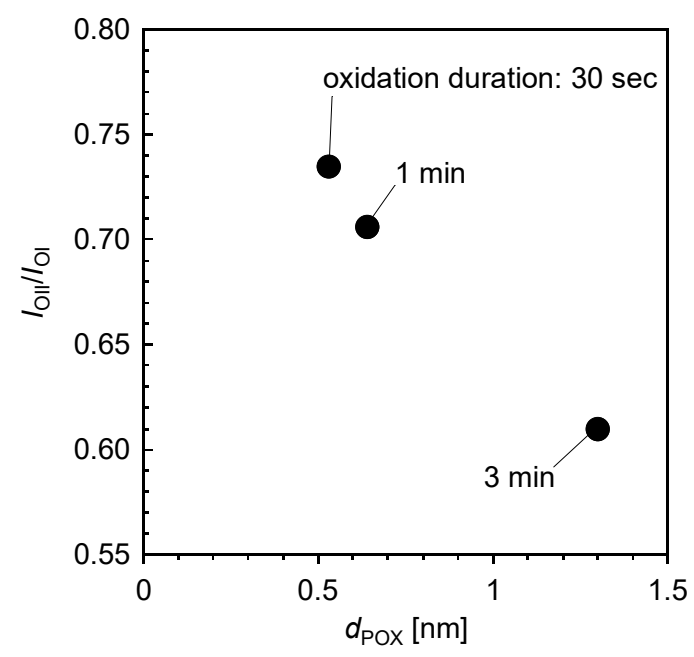

Fig. 7 

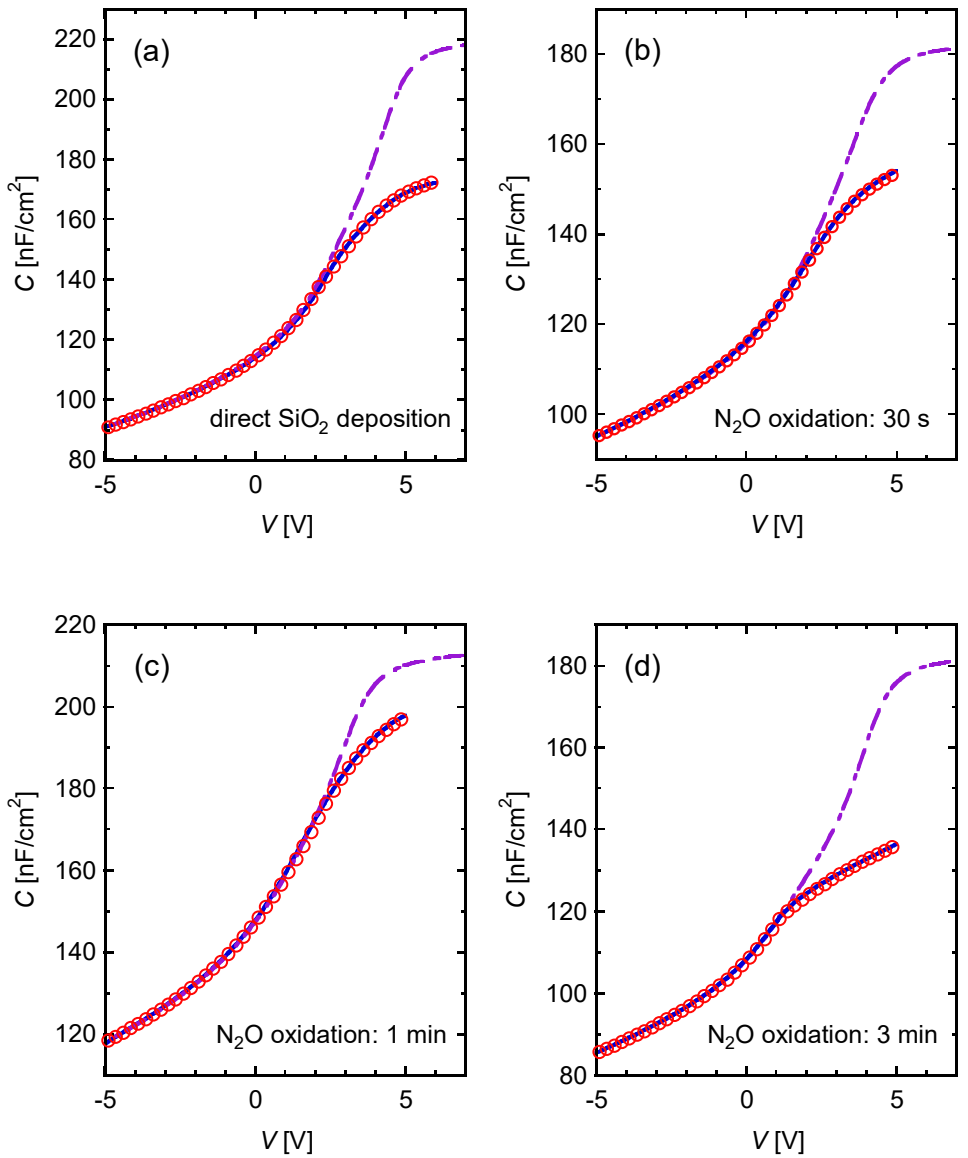

Fig. 8

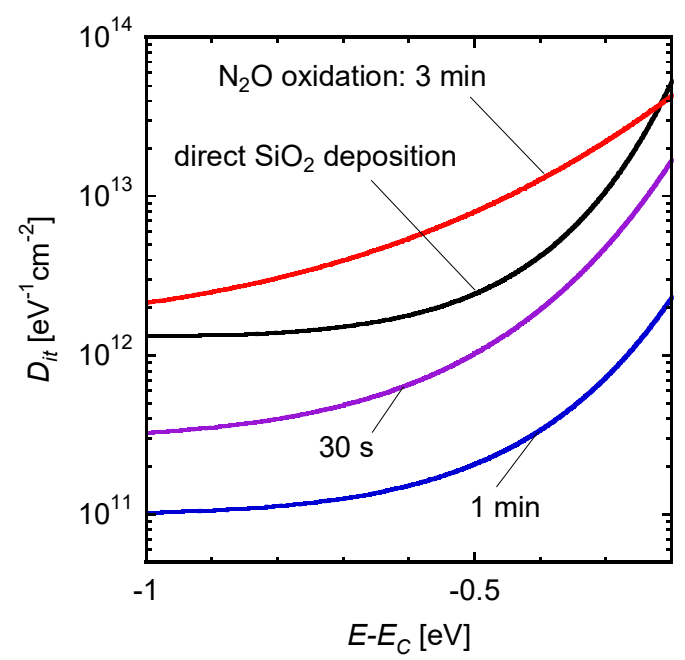

Fig. 9 


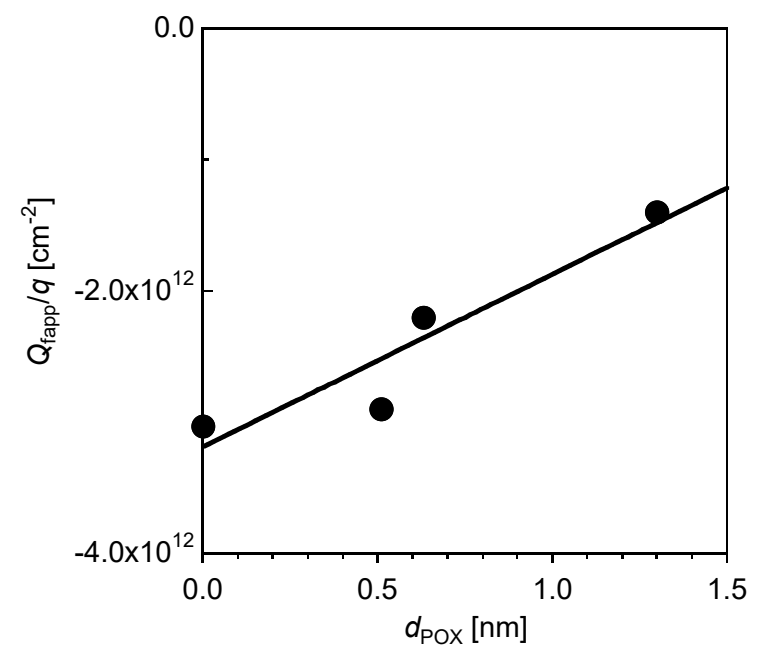

Fig. 10 